\title{
The sparkling Universe: Clustering of voids and void clumps
}

\author{
Marcelo Lares ${ }^{\star}$, Andrés N. Ruiz, Heliana E. Luparello, Laura Ceccarelli, Diego Garcia \\ Lambas \& Dante J. Paz \\ Instituto de Astronomía Teórica y Experimental (CONICET-UNC) and Observatorio Astronómico (UNC), Laprida 854, X5000BGR, Córdoba, Argentina.
}

Accepted XXX. Received XXX; in original form XXX

\begin{abstract}
We analyse the clustering of cosmic voids using a numerical simulation and the main galaxy sample from the Sloan Digital Sky Survey. We take into account the classification of voids into two types that resemble different evolutionary modes: those with a rising integrated density profile (void-in-void mode, or R-type) and voids with shells (void-in-cloud mode, or S-type). The results show that voids of the same type have stronger clustering than the full sample. We use the correlation analysis to define void clumps, associations with at least two voids separated by a distance of at most the mean void separation. In order to study the spatial configuration of void clumps, we compute the minimal spanning tree and analyse their multiplicity, maximum length and elongation parameter. We further study the dynamics of the smaller sphere that encloses all the voids in each clump. Although the global densities of void clumps are different according to their member-void types, the bulk motions of these spheres are remarkably lower than those of randomly placed spheres with the same radii distribution. In addition, the coherence of pairwise void motions does not strongly depend on whether voids belong to the same clump. Void clumps are useful to analyse the large-scale flows around voids, since voids embedded in large underdense regions are mostly in the voidin-void regime, were the expansion of the larger region produces the separation of voids. Similarly, voids around overdense regions form clumps that are in collapse, as reflected in the relative velocities of voids that are mostly approaching.
\end{abstract}

Key words: cosmology: observations - large scale structure of Universe

\section{MOTIVATION}

The structure of the mass distribution in the Universe at large scales can be described as a network with a typically filamentary structure, which intersect forming even denser clumps, the preferred places where galaxy clusters are formed. The origin and evolution of this arrangement can be understood in the framework given by the cosmological models, and supported by observational evidence (e.g. Liddle 2003; Dodelson 2003) which depict nearly the same picture of hierarchical structure formation (Padmanabhan 1993; Peebles 1993). Among the currently discussed scenarios in the literature, the concordance $\Lambda \mathrm{CDM}$ is the preferred model at the present, given its ability to predict a large variety of observed phenomena (Weinberg 2008). As the Universe evolves, mass is accreted onto the densest concentrations, giving rise to large empty regions in the distant future (Dünner et al. 2006; Araya-Melo et al. 2009; Pearson 2015). A different picture of the same process is obtained by looking at the initial low density fluctuations that become increasingly emptier, larger and rounder, as mass flows towards dense regions. These two pictures are complementary and manifest in the largescale distribution of matter (Einasto et al. 1986, 1997), producing a filamentary-void network (e.g. Matsuda \& Shima 1984; Way et al.

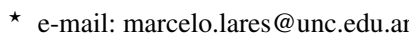

2011; Icke \& van de Weygaert 1991; Leclercq et al. 2015). According to this scenario, not only large-scale flows of mass play a key role in shaping the largest structures, but also they are intimately connected to the mass distribution itself. This reflects the reciprocal action between the source of gravity and the forces it produces, as described by the field equations of general relativity. The scales at which this action can be detected are considerable large (Watkins et al. 2009; Feldman et al. 2010; Nusser et al. 2011; Turnbull et al. 2012). According to this model, large-scale flows of mass play a key role in forming the largest structures and shaping the cosmic web, and its effects can be detected up to considerable large scales (Frisch et al. 1995; Watkins et al. 2009; Feldman et al. 2010; Nusser et al. 2011; Turnbull et al. 2012).

In Lambas et al. (2016) we reported the motions of cosmic voids as a whole, which also show a strong coherence pattern associated to the void velocity field up to large cosmological scales, both in simulations and observations. This effect strongly depends on the type of void considered, with a void-in-void and void-incloud classification scheme proposed by Sheth \& van de Weygaert (2004) that distinguish the internal dynamical behaviour (Paz et al. 2013) according to their environment (Ceccarelli et al. 2013). The coherence pattern in the relative velocities is twofold once voids are divided according to this classification: void coherent bulk velocities define a bimodal dynamical population of mutually attracting 


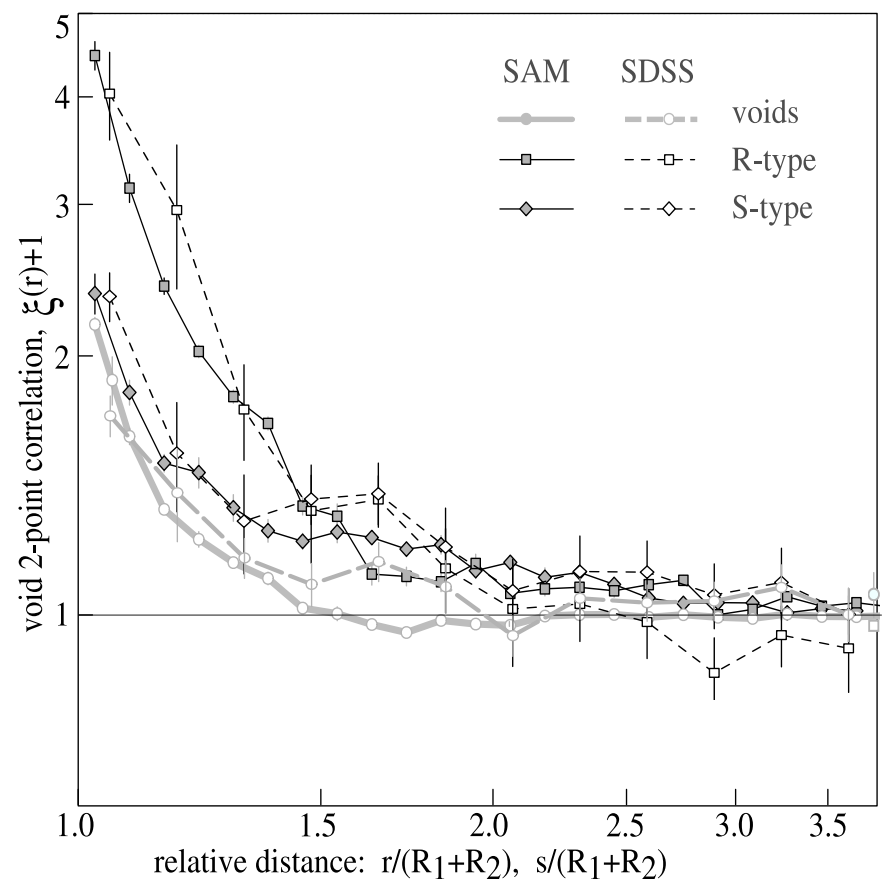

Figure 1. Autocorrelation functions of voids, using the full sample (grey lines), S-type voids (diamonds) and R-type voids (squares), for simulation (solid lines, filled symbols) and SDSS data (dashed lines, empty symbols). Distances are in units of $h^{-1} \mathrm{Mpc}$, in real space for the simulation and in redshift space for SDSS data. Error bars correspond to uncertainties computed by means of Jackknife resampling.

(for shell like voids) or receding (for voids embedded on largescale underdensities) systems. We argue that these global motions contribute to imprint large scale cosmic flows that will shape the formation of structures in the distant future.

In what follows, we recap the properties of the catalogues of voids and study the clustering of voids by computing the 2point autocorrelation function of voids (Sec. 3). Given the significant correlations between voids, a percolation algorithm is suitable to identify conspicuous groups of voids. We then analyse these groups, which we call "void clumps", through their geometrical and dynamical properties (Sec. 4). The dynamical behaviour of void clumps is not equivalent to other regions of the same volume centred in random locations (Sec. 5). Thus, we explore the global dynamics of the regions occupied by the clumps and their internal motions of mass as a function of the type of voids which compose the clumps. Finally, in the Sec. 6, we discuss the results in the context of the hierarchical structure formation scenario.

\section{DATA}

In this section we describe the galaxy catalogues, both observed and simulated, and the void identification algorithm used in this work.

\subsection{Galaxy catalogues}

We use the Main Galaxy Sample of the Sloan Digital Sky Survey Data Release 7 (SDSS-DR7, Abazajian et al. 2009). This sample counts with nearly a million of galaxies with spectroscopic measures, redshifts up to $z \leqslant 0.3$ and an upper apparent magnitude in the $r$-band of 17.77. For this Main Sample, we select galaxies with a limiting redshift $z=0.08$ and a maximum absolute magnitude in the $r$-band of $M_{r}-5 \log _{10}(h)=-19.1$. This guaranties a volume complete galaxy sample at that redshift. The SDSS velocity field we consider, corresponds to the peculiar velocity field derived by Wang et al. (2009, 2012), where the authors employed the linear theory relation between mass overdensities and peculiar velocities to reconstruct the 3D velocity field of galaxies. A detailed analysis of the effects of the linearised velocity field in estimating void bulk velocities in the observational sample can be seen in the Appendix of Ceccarelli et al. (2016).

We also use the semi-analytical galaxies presented by Guo et al. (2011), which were constructed by applying the Munich semianalytic model (SAM) of galaxy formation to the dark matter only Millennium Simulation (MS, Springel et al. 2005). The MS counts $2140^{3}$ dark matter particles evolved from $z=127$ to $z=0$ in a cubic comoving volume of $\left(500 h^{-1} \mathrm{Mpc}\right)^{3}$. The cosmological parameters used in the MS correspond to a $\Lambda \mathrm{CDM}$ flat cosmology with $\Omega_{\mathrm{m}}=0.25, \Omega_{\Lambda}=0.75, \Omega_{\mathrm{b}}=0.045, \sigma_{8}=0.9, h=0.73$ and $n=1.0$. This parameters are consistent with the WMAP1 results (Spergel et al. 2003). The galaxy catalogue of Guo et al. (2011) is public available at the Millennium Database ${ }^{1}$.

In order to make a fair comparison between observations and the simulated data, we analyse SAM galaxy samples with the same number density than the observed galaxy distribution (see e.g. Contreras et al. 2013, 2015). Using the same magnitude cut in the SAM galaxies than in SDSS does not guaranties the same number density, because of the differences between their luminosity functions. Instead, we select all SAM galaxies brighter than $M_{r}-5 \log _{10}(h)=$ -19.7 , which guarantees the volume density needed.

\subsection{Void identification}

The identification of voids was performed following the procedures described in Ruiz et al. (2015), which is a modified version of the algorithms presented in Padilla et al. (2005) and Ceccarelli et al. (2006).

The identification starts using the galaxy catalogues as tracers of the density field and constructing a contrast density field estimation using a Voronoi tessellation, selecting as void candidates all the underdense cells with a density contrast bellow -0.8 . We identify voids both in a numerical simulation and in the SDSS data. Centred in these underdense cells, we compute the integrated density contrast $\Delta(r)$ at increasing values of radius $r$, and select as void candidates the largest spheres which satisfy the condition $\Delta\left(R_{\text {void }}\right)<-0.9$, with $R_{\text {void }}$ as the void radius. In order to recentre each candidate, the centre position is randomly shifted and the procedure described previously is repeated in this new centre, allowing the sphere to grow in size. Finally, a void of radius $R_{\text {void }}$ is selected as the largest sphere satisfying the underdense condition that does not overlap any other underdense sphere. In the case of SDSS data, we restrict our void definition to spherical regions with a fixed global density, excluding those spheres that are not completely within the survey mask. The final catalogues comprise 252 voids for the SDSS sample and 4015 voids for the full Millennium box, both with similar radii distributions in the range 6-24

\footnotetext{
${ }^{1}$ http://gavo.mpa-garching.mpg.de/Millennium
} 

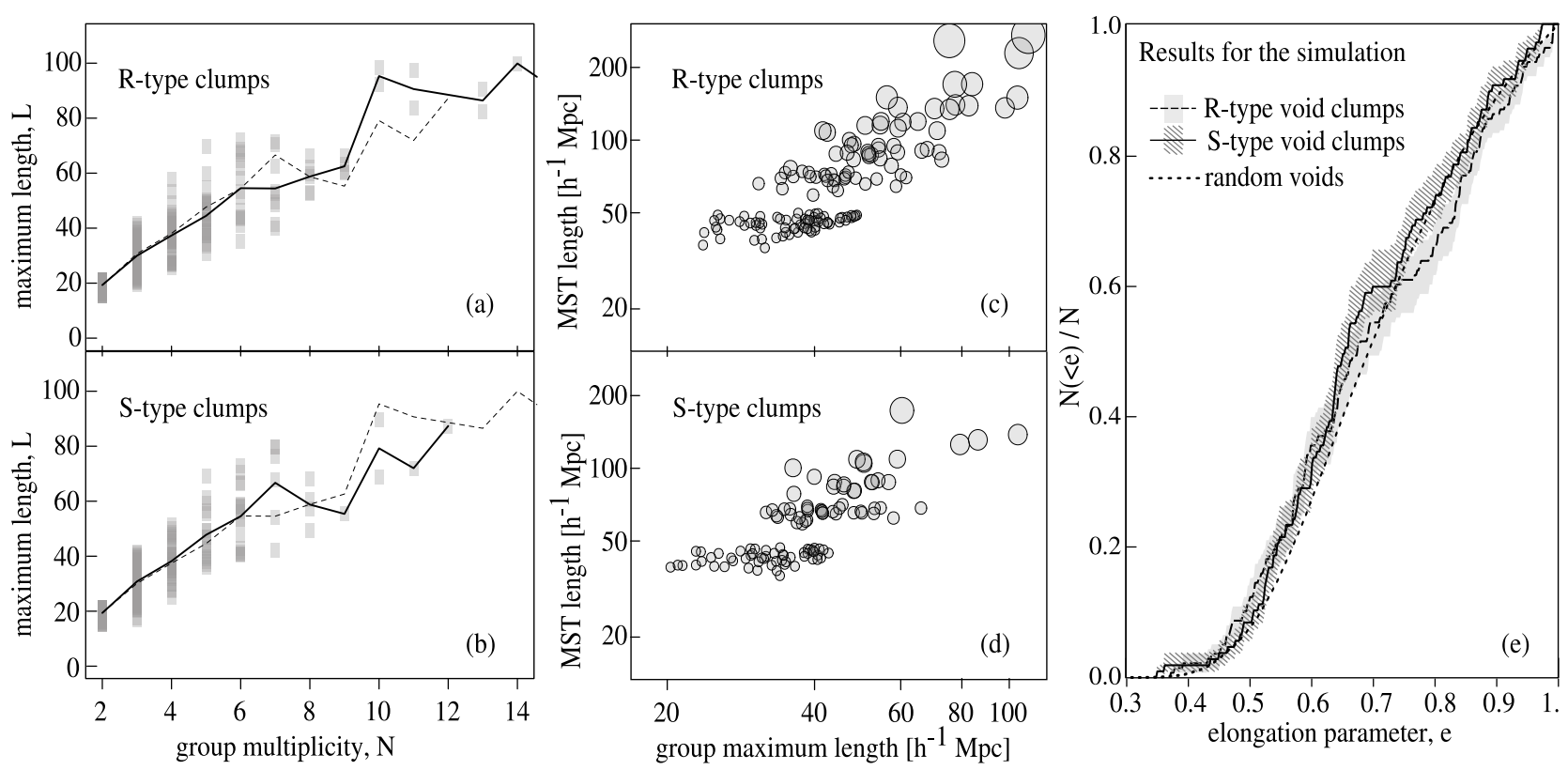

Figure 2. Left: Relation between the maximum separation of voids within a clump and clump multiplicity, for R-type void clumps (a) and S-type void clumps (b). Each grey square corresponds to one group. The means of maximum lengths for each multiplicity are also shown in solid lines, and repeated on both panels for comparison (dashed lines). Centre: Relation between the length of the minimal spanning tree (MST) and the maximum length for R-type void clumps (c) and S-type void clumps (d). The circle size is proportional to the clump multiplicity. Right: Empirical cumulative distributions of the elongation parameter, for R-type (dark solid lines) and S-type void clumps (dark dashed lines). Dotted line corresponds to the distribution for groups of random points with the same multiplicities than void clumps.

$h^{-1} \mathrm{Mpc}$. The larger number of voids in the Millennium box simulation is consistent with the volume difference with the SDSS data.

\section{VOID CLUSTERING}

As mentioned in Sec. 1, the gravitational growth of the large scale structure can be described by means of two complementary scenarios, the accretion of mass onto massive objects and the expansion of underdense regions. In this context, we analyse here the spatial distribution of voids focusing on its relation to large scale flows. Previous results have shown a coherent velocity field of voids (Lambas et al. 2016), an effect that can be explained in terms of the large-scale surrounding distribution of mass (Ceccarelli et al. 2016). Therefore, it is natural to expect a relation between the clustering of mass, large-scale velocity flows, and the clustering of underdense regions.

It must be taken into account that the void definition used in our study comprises regions that contain at most the 10 per cent of the mean density of the Universe. The clustering of voids, as analysed here, is thus a manifestation of the locations of almost empty regions, and has a valuable information from a cosmological perspective since it will eventually allow to study the volumes, shapes, percolation and density profiles of the largest underdense regions at a fixed degree of underdensity.

The correlation function, $\xi(r)$, measures the probability excess of finding a pair of objects at a given relative distance $r$ with respect to a random distribution. This tool has been extensively used to quantify the clustering of galaxies (Einasto et al. 1997; Kerscher et al. 2000) and is a key observable to distinguish cosmological models and test the structure formation scenarios (Matsubara 2004). In a pioneer work, Padilla et al. (2005) examined the void-void correlation function in a numerical simulation. They compared the clustering of haloes and galaxies finding larger voids to be strongly clustered. However the correlation amplitude is not statistically significant due to the small simulation box. Similarly, Clampitt et al. (2016), using the autocorrelation function of SDSS and simulated voids, also find a stronger signal for the larger voids. There are other algorithms to identify voids that are not restricted to the spherical condition. A usually adopted algorithm is the ZOBOV finder (Neyrinck 2008), which makes a Voronoi tessellation of the space to estimate de density field. Voids are identified through a watershed algorithm, as regions around local minima limited by ridges in the density field that separate different minima. The clustering of ZOBOV voids has been analysed in simulations (Hamaus et al. 2014b; Chan et al. 2014; Hamaus et al. 2014a). Hamaus et al. (2014b) analyse the void bias and describe two different populations: small voids, which are overcompensated by the mass in the surrounding regions, and large, undercompensated voids. While small voids have large bias with respect to the dark matter distribution, larger voids are preferentially anti-correlated. The authors argue that the high bias in small voids is due to an overcompensation of void shells around small voids, that are typically voidsin-clouds. This resembles the S-type classification we use in our work, although we find that while most of the large voids are of Rtype, small voids can be of either type in nearly equal number (see Ceccarelli et al. 2013). In spite of these differences, this classification is also based on the spirit of the void-in-cloud (overcompensated) and void-in-void (undercompensated) types and results in different dynamical evolution and different bias parameters. However, Hamaus et al. (2014b) show that the distribution of voids is dominated by a Poisson noise for small voids, and with smaller power for large voids given by the exclusion effects. Hamaus et al. (2014a) expand these ideas and use the void clustering statistic in a cosmological simulation to probe the cosmic expansion history 

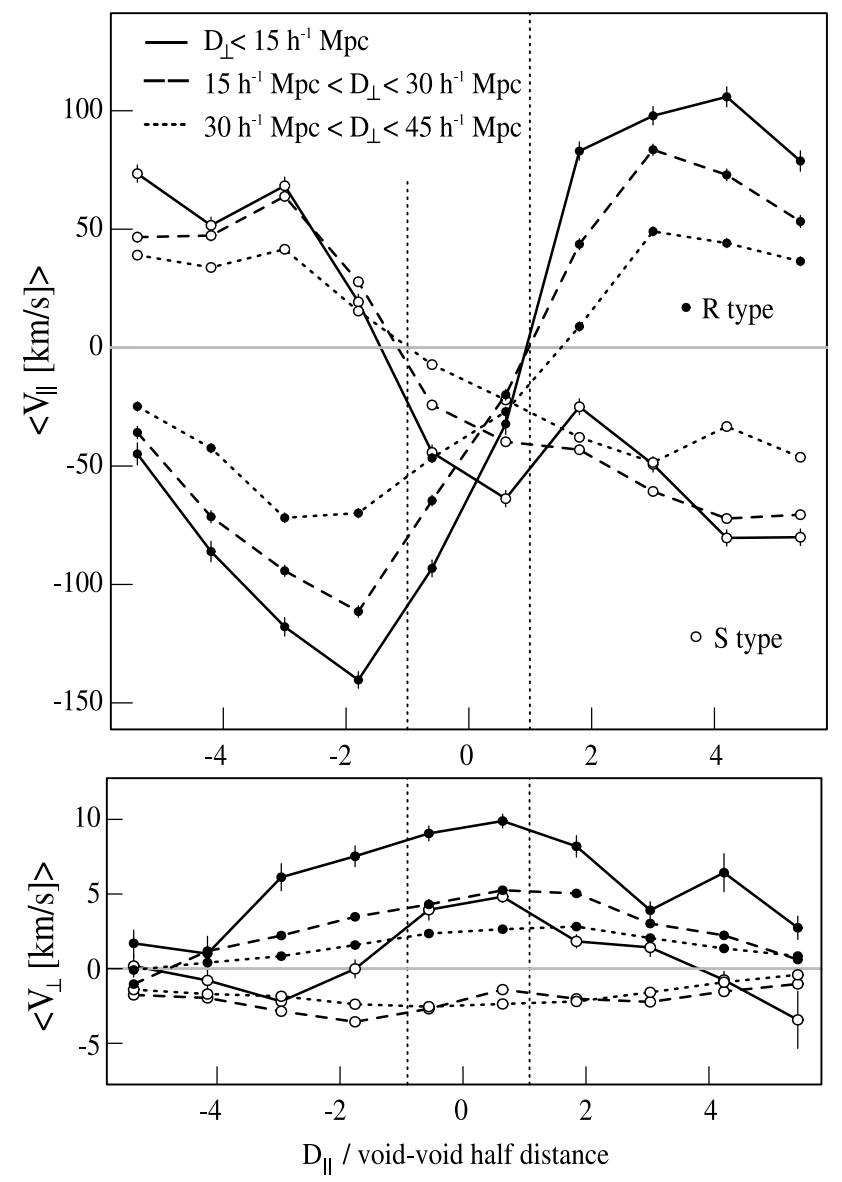

Figure 3. Mean velocity components of SAM galaxies in the directions parallel (upper panel) and perpendicular (bottom panel) to the direction between the two void centres, in clumps formed by two R-type voids (filled circles) or two S-type voids (empty circles). The different curves (see figure key) correspond to galaxies within a perpendicular separation $\mathrm{D}_{\perp}$ range to the axis along the two void members (i.e. cylindrical shells). Vertical dotted lines indicate the position of each void in the pair.

of the universe. These features could be explained by an excursion set formalism (Chan et al. 2014). Zhao et al. (2016) use a different void finder based on a Delaunay triangulation of a set of tracers (DIVE) and identify two different populations of voids characterised by their radii, which resemble the void-in-void and void-in-cloud regimes. The authors compute the power spectrum of DIVE voids and find that, on large scales, large voids show a low bias while small voids are strongly biased, in agreement with previous results. The clustering of voids has also been analysed by Liang et al. (2016), where the authors find a conspicuous signal at nearly $100 h^{-1} \mathrm{Mpc}$ which indicates the presence of the Baryon Acoustic Oscillations of mass in the early Universe. The definition of voids in their study does not account for the so called exclusion effect, i.e., all overlapping underdense spheres are considered. The authors also find a scale dependent bias for different samples of voids depending on void radius, with the larger voids showing the strongest signal. This favours a larger clustering signal for voids in the void-in-void regime. This result was confirmed later by Kitaura et al. (2016), who analyse overlapping density troughs of the density field and argue that the detection of baryonic acoustic oscillations is not significant for the classical definition of disjoint voids.
In this work, we use the correlation function of the distribution of distances between the centres of pairs of voids to measure the degree of clustering of voids. In order to compute this function, we counted pairs in bins of relative distance, that is the comoving separation between void centres divided by the sum of its radii. Here, a separation of $r /\left(R_{1}+R_{2}\right)=1$ means that the pair of voids are in contact, with a separation between centres equal to the sum of their radii. The normalization of the correlation function to the sum of the void radii is convenient since the signal would have a mixture of different contributions from small and large voids in natural units of distance. To construct these functions, we generated mock catalogues in the same box than that of the simulation, using a simple sequential inhibition algorithm to reproduce the exclusion effect produced by the finite size of the voids. This is important since the scales of interest, where the correlation signal is observed, is comparable to the size of the voids. The results, applied to our sample of voids, are shown in Fig. 1 for several samples of voids in the simulation and in the SDSS galaxy catalogue. We compute $\xi(r)$ (simulation) and $\xi(s)$ (observations) for relative distances larger than the unity, due to the exclusion imposed in the identification algorithm (see Sec. 2.2). The dashed (solid) grey line, show the results for the autocorrelation of voids identified on the SDSS (SAM catalogue). Beyond roughly a relative distance of 2 , the distribution of pairs of voids is consistent with a Poisson distribution. On the other hand, there is a range of distances with a significant excess of void pairs, for a typical void size of $10-12 h^{-1} \mathrm{Mpc}$ this corresponds to scales between 20 and $45 h^{-1} \mathrm{Mpc}$. This scale is larger than that of the void shells reported in Paz et al. (2013).

In order to explore the role of the environment on the correlation, we separated voids according to the criteria presented in Ceccarelli et al. (2013), that in turn follows the ideas proposed by Sheth $\&$ van de Weygaert (2004). Voids that have a steep integrated density profile resembling a shell-like surrounding structure are classified as S-type, while voids with a gently rising profile are classified as R-type. This classification was used in previous works (Ceccarelli et al. 2013; Paz et al. 2013; Ruiz et al. 2015; Lambas et al. 2016; Ceccarelli et al. 2016), where it was proved effective at separating two distinct populations of void environments. In Fig. 1 we show the autocorrelation of R-type (squares) and S-type (diamonds) voids for SDSS (dashed lines) and SAM data (solid lines). Remarkably, the autocorrelation of R-type voids is significantly higher than that of the general population within $r \gtrsim 2\left(R_{1}+R_{2}\right)$, indicating that this type of voids are preferentially clustered. At distances larger than $r \gtrsim 2\left(R_{1}+R_{2}\right)$, there is no evidence of a significant difference between the sample populations. This is related to the relatively small volume of the survey data, which gives large uncertainties as indicated by the error bars of the lines corresponding to SDSS galaxies. Similarly, but with lower significance, S-type voids tend to be more clustered than the general void population.

We compute the statistical uncertainties of the correlation on each distance bin using Jackknife or leave-one-out resampling. For a sample of voids with size $N_{v}$, we use the "natural" estimator (Kerscher et al. 2000) of the correlation function, which estimates the correlation function $\xi(i)$ in terms of the number of void pairs $(D D(i))$ and the number of random void pairs $(R R(i))$ at the i-th distance bin that spans from $r_{i}$ to $r_{i+1}$. The central tendency measure of $\xi(i)$ is given by the Jackknife average (Efron 1987; Lupton 1993):

$\bar{\xi}_{J}(i)=\frac{1}{N_{v}-1} \sum_{j=1}^{N_{v}} \xi_{[j]}(i)$, 


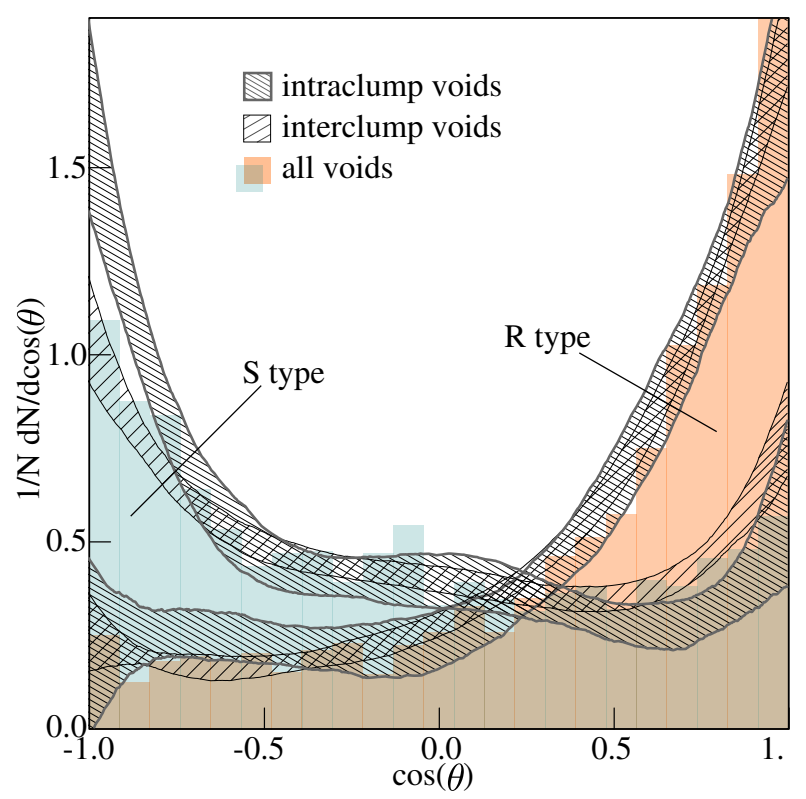

Figure 4. Number density of void pairs as a function of $\cos (\theta)$ for all voids in the the simulation. We show separately the estimations of the distributions of $\cos (\theta)$, with histograms for all $\mathrm{R}$ - and $\mathrm{S}$-type voids and with smoothed curves for voids within the same clump or in different clumps (see figure key). The shaded regions are estimations of the distributions after a dimensionality reduction with a Fourier expansion and represent 1$\sigma$ bootstrap uncertainties. The results for R-type voids and S-type voids are shown separately, where S-type distributions favour negative values of $\cos (\theta)$, corresponding to approaching pairs, and $\mathrm{R}$-type voids are dominated by positive values, corresponding to receding pairs.

where $\xi_{[j]}(i)$ is the $\mathrm{j}$-th Jackknife realization, i.e., the value of $\xi$ computed by leaving the $\mathrm{j}$-th element element out. The number of Jackknife realizations is equal to the number of voids in the sample. For a Jackknife realization the data-data pair counts turn to be $D D_{[j]}$. The random-random pairs $<R R(i)>$ are not affected by the Jackknife step since we use the average of a large number of void sample mocks. Then, the correlation function estimation for the $\mathrm{j}$-th Jackknife realization is given by:

$\xi_{[j]}(i)=\frac{D D_{[j]}(i)}{<R R(i)>} \frac{N_{v}}{N_{v}-1}-1$.

The compensation factor $N_{v} /\left(N_{v}-1\right)$ compensates for the different number of centre voids in the centre-tracer scheme of pair computations. Since it is an autocorrelation and the random samples are generated with the same number of voids for each void sample, the number of tracer is the same and no other correction is required. Finally, the uncertainty is estimated by:

$\hat{\sigma}_{J}^{2}(i)=\frac{N_{v}-1}{N_{v}} \sum_{j=1}^{N_{v}}\left(\bar{\xi}_{[j]}(i)-\bar{\xi}_{J}(i)\right)^{2}$

\section{CLUMPS OF VOIDS}

In this Section we present the definition and identification procedure used to detect void clumps both in the simulation and SDSS data. Also we present an analysis of its geometrical properties.

\subsection{Identification procedure}

The detection of clustering in the void distribution (as shown in Sec. 3) leaded us to apply a percolation algorithm in order to isolate groups of voids, and obtain insight on the origin of the higher correlation observed for the two void types. We searched for groups of voids by implementing the friend-of-friends algorithm. This is a simple algorithm that links together all voids that have at least a subset pair having centres closer than a given linking length, hereafter $\ell$. The numerical value of this length, however, depends on the nature of the problem and must be determined. For both samples ( $\mathrm{R}$ - and S-type voids) we start by considering the mean inter-void separation $\ell_{M V S}$ :

$\ell_{M V S}=\left(\frac{3}{4 \pi n}\right)^{1 / 3}$

where $n$ is the number density of a given void sample. This value can be interpreted as the radius of a sphere placed at random containing on average one void centre. For the R-type sample of voids we obtain a value $\ell_{M V S}=21.64 h^{-1} \mathrm{Mpc}$, whereas for S-type voids $\ell_{M V S}=21.47 h^{-1} \mathrm{Mpc}$ is obtained. Then, we explored several values of the linking length parameter $\ell$ as a variable fraction $f$ of $\ell_{M V S}$ (i.e. $\ell=f \ell_{M V S}$ ). For each case we computed the multiplicity function, i.e., the distribution of multiplicities of the resulting groups. By taking values of $f<1$, a larger abundance of S-type void clumps are obtained in comparison to the R-type clumps for the whole range of multiplicities. On the other hand, for $f>1$ the opposite behaviour is observed, namely, multiplicities are larger for R-type void clumps. Therefore, we defined R-type and S-type void clumps as friend-of-friends groups with a linked length equal to $\ell_{M V S}$, which preserves similar multiplicity distributions for both samples.

\subsection{Properties of void clumps}

We study the geometrical properties of the void clumps by estimating their size and shape. Since the number of members of the groups is typically low, usual methods to compute the shape tensor do not work (Paz et al. 2006). Instead, we computed the minimal spanning tree (hereafter MST, Kruskal 1956) of the centres of voids in each group, and compared it to the maximum separation between any two members. The MST is the graph of minimal length which connects all members in the group. If the group is very elongated, the numerical values of both measures are similar, otherwise, the length of the MST is larger than the maximum length separation

Panels (a) and (b) of Fig. 2 show the maximum length, L, defined as the maximum separation between any pair of void centres that belong to the same void clump, as a function of group multiplicity, for R-type and S-type void clumps respectively, These results are repeated in both panels (dashed lines) in order to allow comparison among void types. Each grey square corresponds to a clump. Solid lines show the mean of the maximum lengths for each multiplicity value. As expected, more populated clumps tend to be larger, with no significant difference between the two types of voids.

Another estimator of the size of a void clump is the length of the MST, which is the sum of its edge longitudes. In panels (c) and (d) of Fig. 2 we show the MST length for R-type and Stype void clumps, respectively, as a function of L. The size of the filled circles is proportional to the multiplicity of the void clump. All triplets have an MST length of order $40 h^{-1} \mathrm{Mpc}$, although the 
maximum length ranges from $20 h^{-1} \mathrm{Mpc}$ to $40 h^{-1} \mathrm{Mpc}$. Similarly, more populated clumps have a larger range of values of the maximum length than in the MST length, which is also verified for both types of void clumps for a fixed multiplicity. However, when comparing the $\mathrm{R}$-type and $\mathrm{S}$-type cases, there is a slight excess of R-type clumps for maximum lengths over $50 \mathrm{~h}^{-1} \mathrm{Mpc}$ and MST lengths over $100 h^{-1} \mathrm{Mpc}$.

The MST length can be compared to the maximum length to get an insight on the shape of the clump. According to this, an elongation parameter can be defined as the ratio of the maximum separation length and the length of the MST for each clump. In panel (e) of Fig. 2 we show the cumulative distribution of this parameter. The results for clumps of R-type voids are shown in dark solid lines, and for clumps of S-type voids with dark dashed lines. Errors represent 1- $\sigma$ uncertainties from a bootstrap resampling estimation. By applying suitable tests, we find no compelling evidence that the observations and the random case are different, independent of multiplicity.

\subsection{Dynamics of galaxies in two-void clumps}

In this subsection, we analyse the dynamics of SAM galaxies in the region surrounding clumps of $\mathrm{N}=2$. As mentioned in Sec. 2.1, we consider a limited magnitude sample of galaxies in order to match the number density of the SDSS volume limited sample. We define two velocity components: $\boldsymbol{V}_{\|}$, along the line containing the two void centres; and $\boldsymbol{V}_{\perp}$, the projection onto the plane normal to this line. The sign of $\boldsymbol{V}_{\|}$is defined positive in the direction to the largest void (i.e. positive velocities are from left to right). For each clump we also define the system centre as the position in the middle of two void members. For each galaxy we compute the two cylindrical components of its separation vector to the system centre, one component along the line of the two void members, $\boldsymbol{D}_{\|}$, and the other, $\boldsymbol{D}_{\perp}$, as a cylindrical radial component on the plane normal to this line. We stack all clumps with $\mathrm{N}=2$ for each type, and consider the normalized projected distance, $d$, along the direction of the two voids: $d=\left|\boldsymbol{D}_{\|}\right| /$void-void half distance. With this definition, the smallest void is at $d=-1$ and the largest at $d=1$. In Fig. 3 we plot the mean $\boldsymbol{V}_{\|}$(upper panel) and $\boldsymbol{V}_{\perp}$ (bottom panel) for galaxies in $\boldsymbol{D}_{\perp}$ bins (different line types, see key figure), for the R-type (filled circles) and S-type (empty circles) stacked void clumps, as a function of the normalized distance along the parallel direction. Vertical dotted lines indicate the positions of the two void centres. For the case of clumps of two R-type voids, we find parallel components of the velocity being mainly positive for $\boldsymbol{D}_{\|}>0$, and negative for $\boldsymbol{D}_{\|}<0$. This implies an outward flux from the void-pair centre.

For S-type voids, is clear that the flux of halos along the parallel direction behaves very differently than the R-type case. The $\boldsymbol{V}_{\|}$values are negative for $\boldsymbol{D}_{\|}>0$ and positive for $\boldsymbol{D}_{\|}<0$, reflecting an inward motion of galaxies. These effects are less pronounced for the outer regions as seen in the different line types. For both void types, these velocity fluxes are predominantly in the parallel direction, since the perpendicular velocity component, shown in the lower panel, is at most a 10 percent of the maximum parallel velocity. These results are in agreement with those presented in previous works (Lambas et al. 2016; Ceccarelli et al. 2016), where the dynamics of void pairs was analysed accordingly to their environments.

\section{ENVIRONMENT AND VOID CLUMPS MOTIONS}

In this Section we analyse different spatial and dynamical properties of voids in clumps, as well as the global motions of void clumps. We also perform a similar analysis applied to the SDSS data.

\subsection{Dynamics of void pairs within clumps}

In Lambas et al. (2016) we showed that the dynamical behaviour of voids is characterised by a combination of two coherent motions: approaching or receding movements between pairs of voids. This produces a bimodal distribution of the relative velocities that can be represented by the values of the cosines between the two velocity vectors of each pair. This bimodal distribution disappears once the pairs of the same type are considered, giving rise to approaching or receding voids in the void-in-cloud or in the void-in-void cases, respectively. Here we explore the relation between this bimodality and the groups of voids. As in Lambas et al. (2016), we compute for each void its bulk velocity, or simply the void velocity, by taking the average velocity of all galaxies within a shell of 0.8 and 1.2 void radius. This is equivalent to the void bulk velocity computed in the full radii range within the void radius (Ceccarelli et al. 2016; Lambas et al. 2016). We then reproduce the procedure used in that work to compute the angle $\theta$ subtended by the void clump members pairwise velocity $(\Delta V)$ and the void relative separation $(\Delta R)$. In Fig. 4 we show the number density of void pairs as a function of $\cos (\theta)$, for several selections of the void pairs used to compute the angle. The density distribution for the complete sample of void pairs, separated accordingly to its void type, is estimated by computing the histogram of the $\cos (\theta)$ values. These distributions are shown for reference as green shaded histograms in Fig. 4.

The estimation of the distributions for voids within the same clump and for voids in different clumps are shown, for S- and Rtype voids, with the shaded regions. These regions are similar to the histograms, but are computed without using bins, by fitting the empirical cumulative distribution with a Fourier expansion, and filtering the hight frequency components that are produced by the noise in the random sampling (Berg \& Harris 2008). The width of the regions indicate the resampling uncertainty computed for each sample. In the Figure, it can be seen that the bimodal behaviour reported by Lambas et al. (2016) holds for the two subsets of voids pairs, irrespective of them being part of a clump or not, except for a slight difference at the extreme values of $\cos (\theta)$ in the S-type clumps.

\subsection{Dynamics of void clumps}

In this subsection we analyse the global density contrast and the dynamics of the large-scale regions around void clumps. To that end, we use the Minimal Enclosing Sphere (MES) of each clump as an approximation of how much spread is the group of voids. The MES is defined as the smallest bounding sphere that completely includes all the voids in the clump. We chose to use this approach by virtue of its simplicity, which also allows the define a centre and explore the dependence of different properties on the distance to this centre.

We have computed the mean mass density contrast and the total mean velocity of the galaxies inside the MESs considering separately clumps of R-type or S-type voids. For comparison, we have also computed these quantities in spheres located at random 

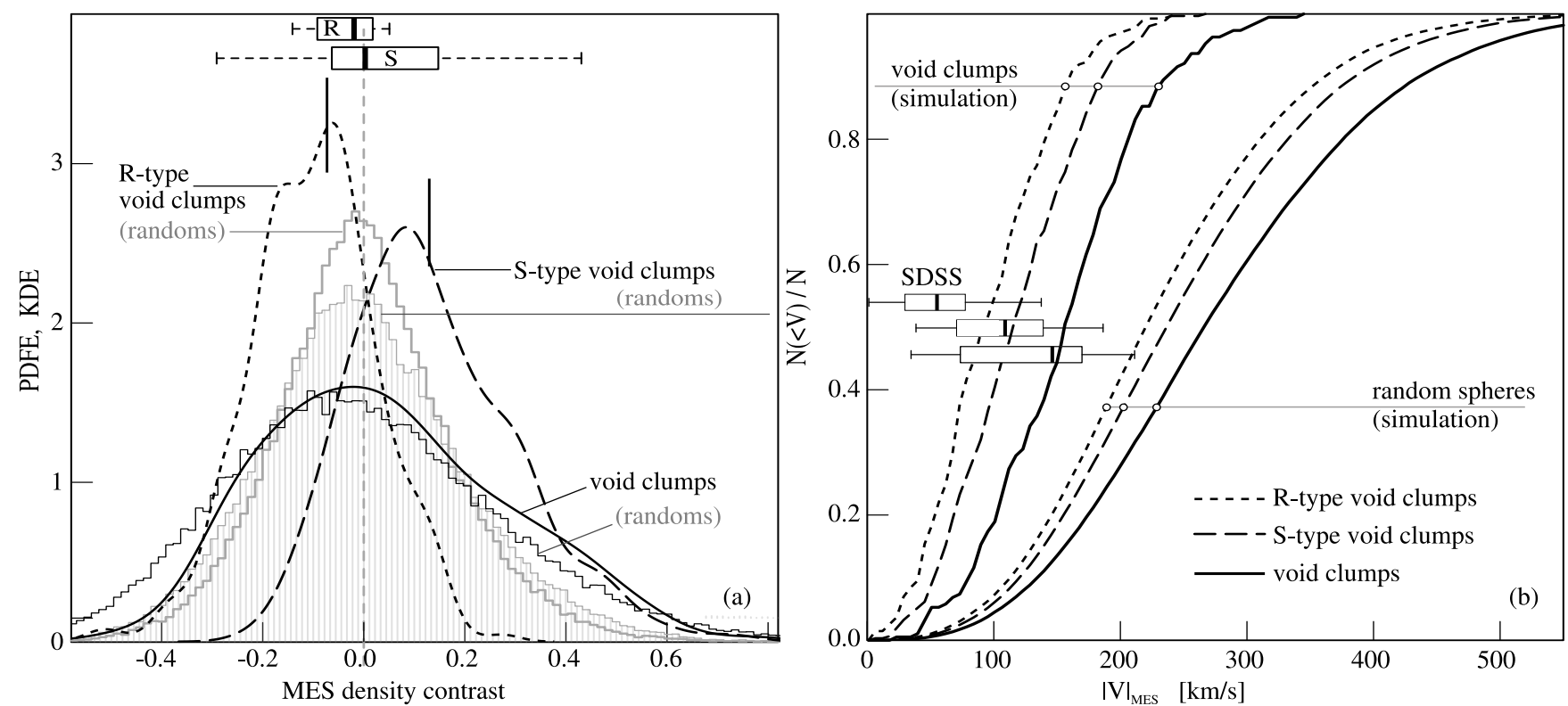

Figure 5. Panel (a): Probability distribution estimates of the density contrast of tracers within the MESs. Thick lines show the kernel density estimates of the distribution of density contrast values for MESs associated to all (solid), R-type (dashed) and S-type (dot-dashed) void clumps. The histograms show the distribution estimates for randomly placed spheres with the same radii distribution than that of the full sample (thin black), R-type (grey), and S-type (grey, vertical dashed) void clumps. The medians of the R/S-types distributions are located with vertical solid lines. The box plots at the top of the panel correspond to R-type and S-type void clumps in the SDSS sample. Panel (b): Empirical cumulative distribution functions of the bulk velocity magnitudes. Curves correspond to the simulation and box plots to the data from SDSS. The three curves on the left correspond to R-type (dotted), S-type (dashed) and the full sample (solid) of void clumps, respectively. The box plots correspond to the same subsets in the sample of SDSS void clumps, according to the one curve that crosses each box. The curves on the right of this panel correspond to samples of randomly placed spheres within the simulation box with the same radii distribution than those in the data with the same line type.

positions, and with the same radii distribution than that of the corresponding sample. In the left panel of Fig. 5 we show the probability distribution estimates of the density contrast of tracers within the MESs. Thick lines show the kernel density estimates (KDE, R Core Team 2016) of the distribution of density contrast values for MESs associated to all (solid), R-type (dashed) and S-type (dot-dashed) void clumps. The vertical marks correspond to the locations of the medians of the R/S-type distributions. The histograms show the distribution estimates for randomly placed spheres with the same distribution of radii than that of the full sample (thin black), R-type (grey), and S-type (grey, vertical dashed) void clumps. According to this figure, the MES density contrast distribution for the complete sample of void clumps is similar to that of random spheres with the same radii distribution. However, when void clumps are split into R/S types, the distributions differ significantly. The distributions of the MES density contrast values of each type of void clumps Individual R- and S-type void clumps have narrower distributions than the full sample and have opposite signs. The fact that a large region containing a number of S-type voids has a positive global density requires the presence of overdense structures between void members of a clump to overcome their low densities. We notice the low number of clumps in the SDSS catalogue given the limited its volume, which makes it difficult a direct comparison of the distributions. The box plots in the top of panel (a) correspond to R- and S-type void clumps in the SDSS sample. These plots are constructed using the median (central mark), the first and third quartiles (borders of the boxes) and the extreme values in the sample (minimum and maximum, indicated by the lines). Although the differences are not very significant, the observations suggest the same trend than the simulation.

In panel (b) of Fig. 5 we show the cumulative distribution functions of the bulk velocity moduli of the simulation void clump MESs. As in panel (a), the box plots correspond to the SDSS data. The three curves on the left correspond to R-type (dotted), S-type (dashed) and the full sample (solid) of void clumps MESs, respectively. The box plots are associated to the same subsets in the sample of SDSS void clumps according to the curve that crosses each box. The curves on the right of this panel correspond to samples of randomly placed spheres within the simulation box with the same radii distribution than those in the data following the same line types. We find results for both $\mathrm{R}$ - and S-type void clumps remarkably similar, showing less than half the bulk velocity of random spheres of similar radii distributions. We stress the observed similarity of R-and S-type void clump MES global motions in spite of their different environments and internal dynamics.

\section{DISCUSSION}

In this work we study the clustering of cosmic voids by computing their autocorrelation function in a cosmological simulation and in a galaxy catalogue. Albeit the different void definitions and identification methods found in the literature, we consider those defined as spheres that have total densities of at most 10 per cent the mean. We explore two different void types characterised according to the shape of their integrated density profiles (Ceccarelli et al. 2013), following theoretical work by Sheth \& van de Weygaert (2004), who introduced void-in-void or void-in-cloud scenarios. We find 
significant differences in the distribution of voids with respect to a Poisson distribution and make use of the $\mathrm{R} / \mathrm{S}$ void type definition to provide further insight on their clustering properties. Following this line, we define clumps of voids as large regions comprising voids of the same type according to the R/S classification. In this work, we look forward to deepen our understanding on the large-scale structure formation through the dynamics of voids. The results obtained for the dynamics of voids are consistent with the sparkling universe picture (Lambas et al. 2016; Ceccarelli et al. 2016), where the large-scale structure growth receives an imprint from void expansions and bulk motions.

Padilla et al. (2005) analysed the clustering of voids identified in a simulation, reporting stronger clustering for the larger voids. This result is consistent with our findings of a higher correlation amplitudes for R-type voids, given their larger average radii (Ceccarelli et al. 2013). Chan et al. (2014) also studied the clustering of voids using numerical simulations, although their definition of voids is different from ours, making it difficult a direct comparison. More recently Clampitt et al. (2016) estimated the correlation of voids in SDSS, obtaining results consistent with our findings. As mentioned in previous sections, we obtain higher correlation amplitudes which can be explained in terms of the environmental classification. The detection of a correlation of larger amplitudes for voids embedded in similar large-scale environments, is a motivation to search for clumps of voids of the same type. Both Rand S-type void clumps have similar geometrical properties, with R-type void clumps slightly more spherical.

Our environmental classification of R/S void types is a natural scenario to study the properties and evolution of voids and their relation to the mass distribution and dynamics. In Paz et al. (2013), we analysed the dynamics of void surroundings using redshift space distortions of the void-galaxy cross-correlations, according to the void environment, finding that large voids are typically in an expansion phase, whereas small ones tend to be surrounded by collapsing overdense regions. This twofold behaviour was observed both in simulations and SDSS data. Clustering and environment of voids have been used to analyse their bias, providing tests for the growth of cosmic structure and measures of cosmological parameters (Hamaus et al. 2015; Chuang et al. 2016; Hamaus et al. 2016). Using redshift space distortions, Achitouv \& Blake (2016) present a test to discriminate between modified gravity models. Hawken et al. (2016) perform a similar study at larger redshifts using VIPERS, obtaining consistent results for the measurement of the linear growth rate. Cai et al. (2016) also use redshift-space distortions around voids and find that the distortion pattern depends on the type of void being considered. The largescale flows of mass induced by this void evolution scenario seem to be an essential part of structure formation, although the effects are limited to the presence of a single void. These previous results are in agreement with the velocity field around void clumps defined by $\mathrm{R} / \mathrm{S}$-type voids, reported in Sec. 5, where clumps of R/S-type voids introduce divergent/convergent large-scale flows. Since clumps of $\mathrm{R} / \mathrm{S}$-type voids are embedded in larger under/overdense regions, these stream motions can be understood as driven by the clump inner mass distribution. The analysis of pairwise velocity of voids in clumps performed reinforces the receding (approaching) processes dominating the relative dynamics of $\mathrm{R}$-type (S-type) voids, although we find voids in different clumps behave similarly. We also analyse the bulk motions of void clumps finding significantly lower velocities than randomly placed spheres with the same radii distribution. In the context of the sparkling universe scenario where voids move in a coherent fashion, void clumps are dynamically conspicuous regions. A low bulk motion has also been reported for watershed voids (Hamaus et al. 2014a; Sutter et al. 2014). It must be noticed, however, that the void clumps described in this work and the voids resulting from the ZOBOV algorithm are of a different nature, and is not straightforward to associate both types of regions. On the other hand, spherical voids have been reported to have non-negligible velocities. This had been suggested by Gottlöber et al. (2003) using spherical voids in a numerical simulation. Also, the bulk velocities are comparable to the velocity of random spheres of the same size (Ceccarelli et al. 2016). Given the different definitions and identification procedures, a direct comparison of the results is not straightforward although their similarity is remarkable.

This work reports on the clustering of voids, as an alternative approach to study the large-scale distribution of mass and its dynamics. The prevalence of a characteristic scale for the clustering of voids allows to define groups of nearby voids, which are suitable laboratories to further explore the implications of the void environments on its evolution and on the formation of the largescale structure of the universe. Galaxies residing in the suburbs of void clumps display dynamical behaviours dominated by velocities toward (away) systems defined by S-type (R-type) of voids. The mean velocities compare well with those derived in Paz et al. (2013) for expanding and collapsing voids and are govern by the global density. All these effects are consistent with the sparkling universe picture (Lambas et al. 2016; Ceccarelli et al. 2016), where the large-scale structure growth can be considered as the result of the voids motions and evolution.

It is worth mentioning that the void statistics is limited by the low number of voids in the observed Universe and this become more restrictive when we consider subsamples of voids according their global density. Besides the observational limitations such as low number of voids, small volume, positions in redshift space and linearized velocities, we obtain, when it is possible perform the comparison, compatible results in theoretical and observational analysis. In the context of the new galaxy surveys such as HETDEX (Hill et al. 2008), Euclid (Laureijs et al. 2011), SDSS-III (Eisenstein et al. 2011), VIPER (Micheletti et al. 2014) and the Dark Energy Survey (Dark Energy Survey Collaboration et al. 2016), the extent of the new data available hold a promising scenario to confront and improve the results on void clustering introduced here.

Given the relevance of void clumps on void dynamics, studying their relation to large structures can shed new light on largescale flows and the formation of the supercluster-void network.

\section{ACKNOWLEDGMENTS}

This work was partially supported by the Consejo Nacional de Investigaciones Científicas y Técnicas (CONICET), and the Secretaría de Ciencia y Tecnología, Universidad Nacional de Córdoba, Argentina. Plots were made using R software and post-processed with Inkscape. This research has made use of NASA's Astrophysics Data System. The authors would like to thank the anonymous reviewer for their valuable suggestions. Funding for the SDSS and SDSS-II has been provided by the Alfred P. Sloan Foundation, the Participating Institutions, the National Science Foundation, the U.S. Department of Energy, the National Aeronautics and Space Administration, the Japanese Monbukagakusho, the Max Planck Society, and the Higher Education Funding Council for England. The SDSS Web Site is http://www.sdss.org/. The SDSS is managed by the Astrophysical Research Consortium for the Participating In- 
stitutions. The Participating Institutions are the American Museum of Natural History, Astrophysical Institute Potsdam, University of Basel, University of Cambridge, Case Western Reserve University, University of Chicago, Drexel University, Fermilab, the Institute for Advanced Study, the Japan Participation Group, Johns Hopkins University, the Joint Institute for Nuclear Astrophysics, the Kavli Institute for Particle Astrophysics and Cosmology, the Korean Scientist Group, the Chinese Academy of Sciences (LAMOST), Los Alamos National Laboratory, the Max-Planck-Institute for Astronomy (MPIA), the Max-Planck-Institute for Astrophysics (MPA), New Mexico State University, Ohio State University, University of Pittsburgh, University of Portsmouth, Princeton University, the United States Naval Observatory, and the University of Washington.

\section{REFERENCES}

Abazajian K. N., et al., 2009, ApJS, 182, 543

Achitouv I., Blake C., 2016, preprint, (arXiv: 1606.03092)

Araya-Melo P. A., Reisenegger A., Meza A., van de Weygaert R., Dünner R., Quintana H., 2009, MNRAS, 399, 97

Berg B. A., Harris R. C., 2008, Computer Physics Communications, 179, 443

Cai Y.-C., Taylor A., Peacock J. A., Padilla N., 2016, MNRAS, 462, 2465

Ceccarelli L., Padilla N. D., Valotto C., Lambas D. G., 2006, MNRAS, 373, 1440

Ceccarelli L., Paz D., Lares M., Padilla N., Lambas D. G., 2013, MNRAS, 434, 1435

Ceccarelli L., Ruiz A. N., Lares M., Paz D. J., Maldonado V. E., Luparello H. E., Garcia Lambas D., 2016, MNRAS, 461, 4013

Chan K. C., Hamaus N., Desjacques V., 2014, Phys. Rev. D, 90, 103521

Chuang C.-H., Kitaura F.-S., Liang Y., Font-Ribera A., Zhao C., McDonald P., Tao C., 2016, preprint, (arXiv: 1605.05352)

Clampitt J., Jain B., Sánchez C., 2016, MNRAS, 456, 4425

Contreras S., Baugh C. M., Norberg P., Padilla N., 2013, MNRAS, 432, 2717

Contreras S., Baugh C. M., Norberg P., Padilla N., 2015, MNRAS, 452, 1861

Dark Energy Survey Collaboration et al., 2016, MNRAS, 460, 1270

Dodelson S., 2003, Modern cosmology. Academic Press

Dünner R., Araya P. A., Meza A., Reisenegger A., 2006, MNRAS, 366, 803

Efron B., 1987, The Jackknife, the Bootstrap, and Other Resampling Plans (CBMS-NSF Regional Conference Series in Applied Mathematics). Society for Industrial Mathematics

Einasto J., Saar E., Klypin A. A., 1986, MNRAS, 219, 457

Einasto J., Einasto M., Frisch P., Gottlober S., Muller V., Saar V., Starobinsky A. A., Tucker D., 1997, MNRAS, 289, 813

Eisenstein D. J., et al., 2011, AJ, 142, 72

Feldman H. A., Watkins R., Hudson M. J., 2010, MNRAS, 407, 2328

Frisch P., Einasto J., Einasto M., Freudling W., Fricke K. J., Gramann M., Saar V., Toomet O., 1995, A\&A, 296, 611

Gottlöber S., Łokas E. L., Klypin A., Hoffman Y., 2003, MNRAS, 344, 715

Guo Q., et al., 2011, MNRAS, 413, 101

Hamaus N., Sutter P. M., Lavaux G., Wandelt B. D., 2014a, J. Cosmology Astropart. Phys., 12, 013

Hamaus N., Wandelt B. D., Sutter P. M., Lavaux G., Warren M. S., 2014b, Physical Review Letters, 112, 041304

Hamaus N., Sutter P. M., Lavaux G., Wandelt B. D., 2015, J. Cosmology Astropart. Phys., 11, 036

Hamaus N., Pisani A., Sutter P. M., Lavaux G., Escoffier S., Wandelt B. D., Weller J., 2016, Physical Review Letters, 117, 091302

Hawken A. J., et al., 2016, preprint, (arXiv: 1611. 07046)

Hill G. J., et al., 2008, in Kodama T., Yamada T., Aoki K., eds, Astronomical Society of the Pacific Conference Series Vol. 399, Panoramic Views of Galaxy Formation and Evolution. p. 115
Icke V., van de Weygaert R., 1991, QJRAS, 32, 85

Kerscher M., Szapudi I., Szalay A. S., 2000, ApJ, 535, L13

Kitaura F.-S., et al., 2016, Physical Review Letters, 116, 171301

Kruskal J. B., 1956, Proc. Amer. Math. Soc., 7, 48

Lambas D. G., Lares M., Ceccarelli L., Ruiz A. N., Paz D. J., Maldonado V. E., Luparello H. E., 2016, MNRAS, 455, L99

Laureijs R., et al., 2011, arXiv:astro-ph/1110.3193

Leclercq F., Jasche J., Wandelt B., 2015, A\&A, 576, L17

Liang Y., Zhao C., Chuang C.-H., Kitaura F.-S., Tao C., 2016, MNRAS, 459,4020

Liddle A., 2003, An Introduction to Modern Cosmology, Second Edition. Wiley

Lupton R. H., 1993, Statistics in theory and practice. Princeton University Press

Matsubara T., 2004, ApJ, 615, 573

Matsuda T., Shima E., 1984, Progress of Theoretical Physics, 71, 855

Micheletti D., et al., 2014, A\&A, 570, A106

Neyrinck M. C., 2008, MNRAS, 386, 2101

Nusser A., Branchini E., Davis M., 2011, ApJ, 735, 77

Padilla N. D., Ceccarelli L., Lambas D. G., 2005, MNRAS, 363, 977

Padmanabhan T., 1993, Cosmology. Cambridge University Press, Cambridge, UK

Paz D. J., Lambas D. G., Padilla N., Merchán M., 2006, MNRAS, 366, 1503

Paz D., Lares M., Ceccarelli L., Padilla N., Lambas D. G., 2013, MNRAS, 436, 3480

Pearson D. W., 2015, MNRAS, 449, 3212

Peebles P., 1993, Principles of Physical Cosmology. Princeton University Press, Princeton

R Core Team 2016, R: A Language and Environment for Statistical Computing. R Foundation for Statistical Computing, Vienna, Austria, https://www.R-project.org/

Ruiz A. N., Paz D. J., Lares M., Luparello H. E., Ceccarelli L., Lambas D. G., 2015, MNRAS, 448, 1471

Sheth R. K., van de Weygaert R., 2004, MNRAS, 350, 517âĂŞ538

Spergel D. N., et al., 2003, ApJS, 148, 175

Springel V., et al., 2005, Nature, 435, 629

Sutter P. M., Elahi P., Falck B., Onions J., Hamaus N., Knebe A., Srisawat C., Schneider A., 2014, MNRAS, 445, 1235

Turnbull S. J., Hudson M. J., Feldman H. A., Hicken M., Kirshner R. P., Watkins R., 2012, MNRAS, 420, 447

Wang H., Mo H. J., Jing Y. P., Guo Y., van den Bosch F. C., Yang X., 2009, MNRAS, 394, 398

Wang H., Mo H. J., Yang X., van den Bosch F. C., 2012, MNRAS, 420, 1809

Watkins R., Feldman H. A., Hudson M. J., 2009, MNRAS, 392, 743

Way M. J., Gazis P. R., Scargle J. D., 2011, ApJ, 727, 48

Weinberg S., 2008, Cosmology. Oxford University Press, Oxford

Zhao C., Tao C., Liang Y., Kitaura F.-S., Chuang C.-H., 2016, MNRAS, 459,2670 